\title{
Efecto de la Encapsulación en Secado por Atomización de Biocomponentes de Pitahaya Amarilla con Interés Funcional
}

\author{
Yessica L. Diaz ${ }^{(1)}$, Laura S. Torres ${ }^{(1)}$, Johanna A. Serna ${ }^{(1) \star}$ y Luz I. Sotelo(2) \\ (1) Universidad La Gran Colombia Seccional Armenia, Facultad de Ingenierías, Programa de Ingeniería \\ Agroindustrial, Armenia, Quindío, Colombia. (e-mail: diazmaryessica@miugca.edu.co, \\ torresvallaura@miugca.edu.co, sernajimjohanna@miugca.edu.co) \\ (2) Universidad de La Sabana, Escuela Internacional de Ciencias Económicas y Administrativas, \\ Gastronomía, Chía, Cundinamarca, Colombia. (e-mail: Indira.sotelo@unisaba.edu.co) \\ ${ }^{*}$ Autor a quien debe ser dirigida la correspondencia
}

Recibido Ene. 26, 2017; Aceptado Mar. 20, 2017; Versión final Jun. 12, 2017, Publicado Dic. 2017

\begin{abstract}
Resumen
La pitahaya amarilla contiene biocomponentes como los polifenoles cuya capacidad antioxidante podría causar un efecto fisiológico más allá del nutricional. Sin embargo, estos biocomponentes son sensibles a condiciones ambientales, de procesamiento, y/o gastrointestinales cuando son consumidos. Por ello, fueron sometidos a encapsulación mediante secado por atomización para evaluar el efecto que tiene dicha técnica sobre su conservación. Los resultados mostraron que el tratamiento con mayor efecto fue el realizado a una temperatura de $150{ }^{\circ} \mathrm{C}$ con $40 \%$ de maltodextrina, logrando prolongar la estabilidad hasta 72 horas para polifenoles totales y 240 horas para la capacidad antioxidante. Con esto se garantiza que la liberación de estos se realice en el intestino delgado, lugar donde quedan disponibles para que el organismo los adsorba.
\end{abstract}

\section{Encapsulation Effect on Spray Drying of Yellow Pitahaya Biocomponents of Functional Interest}

\begin{abstract}
Yellow pitahaya contains biocomponents such as polyphenols with antioxidant capacity that may cause a physiological effect besides the nutritional aspects. However, these biocomponents are sensible to environmental conditions, processing, and/or gastrointestinal environment when they are consumed. Therefore, biocomponents were subjected to encapsulation by spray drying to evaluate the effect of this technique on their conservation. The results show that the most effective treatment was that performed at temperature of $150{ }^{\circ} \mathrm{C}$ with $40 \%$ maltodextrin, achieving longer stability of up to 72 hours for total polyphenols and 240 hours for the antioxidant capacity. In this way, it is guaranteed that release of these biocomponents will occur in the small intestine, where they are available for the body to adsorb them.
\end{abstract}

Keywords: encapsulation; spray drying; bioactive substances; bioavailability; storage stability 


\section{INTRODUCCIÓN}

En los últimos años, la alimentación y la nutrición se han convertido en ejes de investigación constante, permitiendo que hoy en día se hable de "Nutrición Optima", es decir la calidad de los alimentos que se consumen con relación a los nutrientes y beneficios que aportan a la salud, siendo precisamente en este contexto donde surgen los denominados alimentos funcionales, los cuales contienen componentes bioactivos que afectan de manera positiva funciones específicas del organismo, promoviendo un efecto más allá de lo nutricional, contribuyendo a la disminución del riesgo de contraer enfermedades crónicas no trasmisibles (ENT) que, según la World Health Organization WHO (2014) son responsables del $63 \%$ de las muertes que se presentan ya sea por causas metabólicas y fisiológicas o por la influencia de factores de riesgo comportamentales como el tabaquismo, el sedentarismo, el uso nocivo del alcohol y las dietas malsanas, siendo precisamente en este último factor, donde por llevar una mala alimentación, consumiendo elevadas cantidades de sal, grasas saturadas y ácidos grasos trans, se incrementa la hipertensión y cardiopatías; así mismo, la no ingesta de frutas y verduras, aumenta el riesgo de padecer enfermedades cardiovasculares, cáncer de estómago y cáncer colorrectal.

Una forma de contrarrestar y/o prevenir las enfermedades mencionadas, es realizando cambios en los hábitos alimenticios, optando por consumir aquellos alimentos que poseen componentes bioactivos en mayor proporción como los vegetales, entre ellos la pitahaya amarilla (Selenicereus megalanthus Haw), que en sus diferentes partes (pulpa, semilla, cáscara y tallo), contienen biocompuestos como los polifenoles, que reducen la oxidación generada por radicales libres, evitando el deterioro de las células y disminuyendo el riesgo de sufrir ENT tales como enfermedades cardiovasculares, diabetes, cáncer y enfermedades respiratorias; a lo que se ha denominado capacidad antioxidante (Shete et al., 2015). Sin embargo, es preciso tener en cuenta que algunos de estos componentes tienen una durabilidad mínima debido a que reaccionan fácilmente con otros compuestos y se deterioran a altas temperaturas, luz y oxígeno (Pulido et al., 2010); es por ello que se requiere desarrollar medidas que conduzcan a mejorar su conservación.

Un método convencional y económico para disminuir el deterioro de biocompuestos es la encapsulación a través de secado por atomización, caracterizado por atrapar sustancias sensibles dentro de una matriz, formando una barrera que reduce su pérdida, aumenta su estabilidad y mejora su biodisponibilidad (Sharifi et al., 2015). Este método, ha sido utilizado en la protección de flavonoides (Shaaruddin et al., 2017), antioxidantes (Araujo-Díaz et al., 2017; Saikia et al., 2015), probióticos (Chaikham et al., 2017), vitaminas (Carlan et al., 2017) y enzimas (Díaz-Martínez et al., 2016).

No obstante, es preciso tener en cuenta que para llevar a cabo el proceso de encapsulación y evitar inconvenientes relacionados con la aglomeración de partículas, apelmazamiento y pegajosidad que se pueden presentar como consecuencia de la baja temperatura de transición vítrea ( $\mathrm{Tg}$ ) del sistema, atribuida directamente a la poca afinidad química entre las moléculas de agua y los azúcares de bajo peso molecular contenidos en productos alimenticios ricos en azúcar, como es el caso de los zumos de frutas ; es necesario utilizar agentes encapsulantes caracterizados por su alto peso molecular y baja viscosidad, que aumentan la Tg del sistema (Araujo-Díaz et al., 2017), reducen el contenido de humedad, actúan como barrera física para el oxígeno y la luz garantizando la protección y evitando la afectación química y enzimática (Saikia et al., 2015).

Diferentes materiales se pueden emplear como agentes encapsulantes, sin embargo, hidratos de carbono como la maltodextrina, con diferentes equivalentes de dextrosa, es el más utilizado en la conservación de compuestos bioactivos, debido a que es económica, de fácil adquisición y además posee funciones tales como formar soluciones incoloras de baja viscosidad en altas concentraciones, favoreciendo el proceso de secado por atomización y permitir la formación de una película o barrera protectora que reduce la permeabilidad del oxígeno y maximiza la incorporación y retención de compuestos funcionales (Saikia et al., 2015).

Algunos autores han evaluado el efecto de esta técnica sobre la estabilización de las propiedades microestructurales, la distribución de biocomponentes en las micropartículas (Porras-Saavedra et al., 2015), las propiedades fisicoquímicas, reológicas y antioxidantes de diferentes matrices alimentarias tales como cáscara (Ee et al., 2014) y zumos de pitahaya roja y blanca (Kar-Hing et al., 2013) entre otros. Asimismo, se han analizado materiales empleados como agentes de encapsulación, observando potencial coadyuvante en maltodextrina para el secado de aceite de coco (Matsuura et al., 2015), en quitosano para la encapsulación de ácido ascórbico (Pulido et al., 2010) y en alginato de sodio, hidroxipropil-metil-celulosa y lisozima para la microencapsulación de compuestos bioactivos alimentarios (Borgogna et al., 2010). Por otro lado, algunos autores han comparado esta técnica con otras, como es el caso de Ramírez et al. (2012) quienes determinaron el efecto la conservación de polifenoles en un jugo de fruta modelo secado por aspersión y liofilización. 
Es así como en esta investigación se planteó evaluar el efecto de la encapsulación a través de secado por atomización sobre la estabilidad y biodisponibilad de biocompuestos de interés funcional de pitahaya amarilla Selenicereus megalanthus Haw, debido a la escasa información en el estudio del efecto de la encapsulación, utilizando maltodextrina como agente encapsulante, como alternativa para la conservación y aumento de su estabilidad, evaluando variables como: temperatura de secado y concentración de maltodextrina empleada y variables de respuesta: contenido de polifenoles totales, capacidad antioxidante y liberación in vitro de los compuestos fenólicos.

\section{MATERIALES Y MÉTODOS}

A continuación, se describe el tipo de material vegetal empleado en la extracción de biocomponentes así como también la técnica de encapsulación utilizada y los análisis realizados, correspondientes a la evaluación de la estabilidad en el tiempo de componentes fenólicos, su liberación bajo condiciones gastrointestinales simuladas in vitro, el diseño experimental y análisis estadístico.

\section{Material vegetal}

En las determinaciones se empleó pitahaya amarilla (Selenicereus megalanthus Haw) en estado de madurez 6 de acuerdo con la NTC 3554, proveniente de cultivos del municipio de Roldanillo, Valle del Cauca y suministrada por la Asociación de Productores de Pitahaya-ASSOPITAYA.

\section{Adecuación del material vegetal}

Los frutos de la pitahaya amarilla fueron lavados con agua potable, se le retiraron las espinas mediante el uso de un cepillo y se desinfectaron sometiéndolos a un tiempo de exposición de 10 min con Citrosan $\circledast$ (Diken International, México) a una concentración de $0.25 \% \mathrm{~V} / \mathrm{V}$. Luego se obtuvo de forma manual la pulpa con las semillas, utilizadas en la preparación de la solución a encapsular.

La pulpa con las semillas de la pitahaya amarilla se licuó durante 3 minutos, se filtró con lienzo y posteriormente se le adicionó $40 \%$ V/V de agua destilada, consistiendo el jugo utilizado en la preparación de la solución que posteriormente se sometió a encapsulación, determinaciones correspondientes a polifenoles totales y capacidad antioxidante, así como también a la simulación de la liberación in vitro de los biocomponentes (polifenoles totales).

\section{Preparación de la solución a encapsular}

Esta solución, consistió en $100 \mathrm{~mL}$ del jugo de pitahaya amarilla con $13 \pm 0.1^{\circ}$ Brix, mezclado con el agente encapsulante en las concentraciones de 40 y $50 \%$ p/V, estos porcentajes según trabajos previos de los autores, obteniendo disoluciones de $36 \pm 0.2$ y $40 \pm 0.6^{\circ}$ Brix respectivamente.

El material empleado como agente encapsulante fue maltodextrina de maíz con dextrosa equivalente de 17 (Tecnas, Colombia), el cual fue seleccionado por ser considerado como un portador o encapsulante económico y de fácil adquisición en el mercado, que provee características que contribuyen al aumento de la estabilidad de biocompuestos (Souza et al., 2014).

\section{Encapsulación mediante secado por atomización}

La encapsulación se realizó en un equipo de secado por atomización (Lab-Scale Spray Dryer 7614YC-015 Pilotech, China), a ocho condiciones de temperaturas de entrada, una temperatura de salida que se mantuvo fija y a dos concentraciones de agente encapsulante (Tabla 1).

Tabla 1: Condiciones de encapsulación

\begin{tabular}{|c|c|c|c|}
\hline \multirow{2}{*}{ Tratamiento } & \multicolumn{2}{|c|}{ Temperatura de secado $\left({ }^{\circ} \mathrm{C}\right)$} & \multirow{2}{*}{$\begin{array}{c}\text { Concentración de agente } \\
\text { encapsulante }(\% \text { p/V) }\end{array}$} \\
\hline & Entrada & Salida & \\
\hline $\mathrm{T} 1$ & 110 & & 40 \\
\hline $\begin{array}{l}\text { T2 } \\
\text { T3 }\end{array}$ & $\begin{array}{l}120 \\
130\end{array}$ & & $\begin{array}{l}40 \\
40\end{array}$ \\
\hline $\mathrm{T} 4$ & 150 & 40 & 40 \\
\hline T5 & 110 & & 50 \\
\hline T6 & 120 & & 50 \\
\hline $\mathrm{T} 7$ & 130 & & 50 \\
\hline T8 & 150 & & 50 \\
\hline
\end{tabular}




\section{Evaluación de la estabilidad de los biocomponentes fenólicos en el tiempo}

La evaluación de la estabilidad de los biocomponentes presentes en el jugo de pitahaya amarilla fresca y encapsulada, se llevó a cabo mediante la elaboración de una cinética en función del tiempo de almacenamiento, el cual fue realizado sin recubrimiento alguno. Como variables de respuesta se midieron el contenido de polifenoles totales y la capacidad antioxidante, estas metodologías se describen más adelante.

El almacenamiento de los biocomponentes encapsulados se realizó a condiciones de abuso $\left(40 \pm 0.2{ }^{\circ} \mathrm{C}\right.$ y $60 \pm 0.2 \%$ de humedad relativa) en cámara climática (C115 Plus Dies 140801 - Comtitronic, Colombia); como control se utilizó el jugo en fresco de pitahaya amarilla, el cual se almacenó a condiciones ambientales $\left(20 \pm 0.2^{\circ} \mathrm{C}\right.$, y $60 \pm 0.2 \%$ de humedad relativa), debido a que, a las condiciones de abuso, la alteración de los componentes se realizaba a una mayor tasa.

Las mediciones se realizaron en función del tiempo de almacenamiento y de la tasa de cambio de los biocomponentes presente en el jugo fresco (control) y encapsulado, definiendo para la determinación de polifenoles un periodo de medición de $0,2,4,6$ y 24 horas para el control y de 24 horas para los encapsulados. La capacidad antioxidante se evaluó cada 30 minutos en el control y cada 18, 24, 30 y 60 horas en los encapsulados.

Para determinar el tiempo de estabilidad de las muestras evaluadas se tuvo en cuenta lo reportado en el estudio realizado por Posada-Cardona (2011), quien establece que un producto almacenado en cámara climática durante 24 horas a las condiciones antes mencionadas, equivale a 96 horas en condiciones normales $\mathrm{y} / \mathrm{o}$ ambientales de almacenamiento (es decir condiciones de temperatura entre 15 y $30{ }^{\circ} \mathrm{C}$ y humedad relativa entre 55 y $75 \%$ ).

Todos los resultados se analizaron mediante el porcentaje de la variación relativa $(\Delta \mathrm{Y})$ respecto al tiempo utilizando la ecuación (1).

$\Delta \mathrm{Y}=\left(\frac{\mathrm{Y}_{\mathrm{i}}-\mathrm{Y}_{\mathrm{j}}}{\mathrm{Y}_{\mathrm{i}}}\right) * 100$

Dónde $\mathrm{Y}=$ Propiedad (Polifenoles totales y/o capacidad antioxidante) $; \mathrm{i}=$ muestra inicial $; \mathrm{j}=$ muestra final

\section{Cuantificación de polifenoles totales}

El contenido de polifenoles totales se determinó por el método de Folin-Ciocalteu, reportado por Jara-Palacios et al. (2014), para lo que se obtuvo extractos a partir de la mezcla de pitahaya amarilla (pulpa y semilla), utilizado etanol al $80 \%$, con $0.25 \mathrm{~mL}$ de extracto, $0.25 \mathrm{~mL}$ de reactivo de Folin-Ciocalteu (Panreac, USA) y $3.25 \mathrm{~mL}$ de Carbonato de sodio anhidro $-\mathrm{Na}_{2} \mathrm{CO}_{3}$ (Merck, Alemania) al $20 \% \mathrm{p} / \mathrm{V}$; se agitó por $90 \mathrm{~min}$ a $1.26 \mathrm{G}$ (Hettich Universal 32R@, Kirchlengern, Alemania) y se dejó en reposo por 2 horas para cuantificación por espectrofotómetria (Genesys10 UV-VIS Scanning 335906 - ThermoScientific, USA) a $765 \mathrm{~nm}$; los resultados obtenidos son expresados en $\mathrm{mg}$ de ácido gálico (GAE)/100g de muestra seca.

\section{Determinación de la capacidad antioxidante}

Para cuantificar la capacidad antioxidante las muestras encapsuladas se realizó un pretratamiento para liberar los biocompuestos. Una mezcla de $1 \mathrm{~g}$ de muestra en $3 \mathrm{~mL}$ de agua, fue homogenizada en un vortex (G-560, Fisherbrand, USA) por 3 minutos; Se realizó una dilución (V/V) 1:30 con etanol, posteriormente fueron centrifugadas a $1.26 \mathrm{G}$ por 3 minutos a $4^{\circ} \mathrm{C}$ (Hettich Universal $32 \mathrm{R} \odot$, Kirchlengern, Alemania), posteriormente el sobrenadante se le adicionó $1,5 \mathrm{~mL}$ del reacitvo ABTS (azinobis 3-etilbenzotiazolina-6-ácido sulfónico, Sigma-Aldrich, Alemania) y 1,5 mL del reactivo DPPH (2,2-difenil-1-picrilo-hidracilo-hidrato, Sigma-Aldrich, Alemania), las muestras se llevaron a lectura a $732 \mathrm{~nm}$ para ABTS y a $540 \mathrm{~nm}$ para DPPH. En los dos métodos se empleó un espectrofotómetro de luz visible (Genesys10 UV-VIS Scanning 335906 - ThermoScientific, USA). Los resultados obtenidos son expresados en $\mu \mathrm{gmol} g-1$ Trolox eq Los resultados obtenidos son expresados en $\mu \mathrm{gmol} g-1$ Trolox eq y se les evaluó la velocidad global de descenso $\left(v_{\downarrow \mathrm{Y}}\right)$ en función del tiempo utilizando la ecuación (2).

$v_{\downarrow \mathrm{Y}}=\left(\frac{\mathrm{Y}_{\mathrm{i}}-\mathrm{Y}_{\mathrm{j}}}{t}\right)$

Dónde $\mathrm{Y}=$ Propiedad (capacidad antioxidante) $; \mathrm{i}=$ muestra inicial; $\mathrm{j}=$ muestra final; $t=$ tiempo total de almacenamiento 


\section{Liberación in vitro de los biocomponentes fenólicos de la pitahaya amarilla encapsulados}

La liberación in vitro de los biocomponentes presente en las muestras encapsuladas se evaluó siguiendo el protocolo reportado por Fernández-León (2015) con algunas modificaciones. Para ello, fueron simuladas las condiciones gastrointestinales humanas así:

\section{Fase estomacal}

En la fase estomacal, los encapsulados se llevaron a un $\mathrm{pH}$ de 2 con una solución de $\mathrm{HCl}$ al $1 \mathrm{~N}$ (Merck, Alemania); se midió el contenido de polifenoles totales a las 0,2 y 4 horas siguiendo la técnica mencionada anteriormente.

\section{Fase intestinal}

Para simular las condiciones intestinales, se llevaron los encapsulados a un $\mathrm{pH}$ de 7 , ajustado con $\mathrm{NaOH}$ al $1 \mathrm{~N}$ (Merck, Alemania) con adición de las sales biliares (Sigma-Aldrich, Alemania); se midió el contenido de polifenoles totales a las 0,2 y 4 horas siguiendo la técnica mencionada anteriormente.

\section{Diseño experimental y análisis estadístico}

Se realizó un diseño factorial completamente al azar. Los factores evaluados fueron: temperatura de entrada al equipo de secado por atomización y concentración de agente encapsulante. Todas las determinaciones se realizaron por triplicado y los resultados referentes a concentración de polifenoles, capacidad antioxidante y liberación in vitro de los biocomponentes fenólicos, fueron sometidos a un análisis de varianza(ANAVA), con comparación de los valores medios mediante la prueba de Tukey a un nivel de significancia del 95\%, utilizando el software estadístico Statgraphics $5.1^{\circledR}$.

\section{RESULTADOS Y DISCUSIÓN}

Los resultados concernientes a la evaluación de la estabilidad en el tiempo de los biocomponentes fenólicos (polifenoles totales y capacidad antioxidante) y su liberación bajo condiciones gastrointestinales simuladas in vitro, se relacionan a continuación; logrando con estos determinar el efecto que tiene la técnica de encapsulación mediante secado por atomización en la conservación de componentes bioactivos de interés funcional de la pitahaya amarilla.

\section{Evaluación de la estabilidad de los biocomponentes fenólicos en el tiempo}

La estabilidad en el tiempo de los polifenoles y la capacidad antioxidante presente tanto en el control (pitahaya amarilla fresca) como en los encapsulados obtenidos mediante diferentes tratamientos, se expresa a continuación:

\section{Determinación de polifenoles totales}

El contenido de polifenoles totales en el control y en los encapsulados, se muestran en la Tabla 2 y 3 . Los resultados muestran que esta variable presenta mayor concentración en los encapsulados que en el control, lo cual pudo ser debido a que estos biocompuestos se concentraron por la eliminación de humedad o porque la aplicación de calor durante el proceso de secado posiblemente escindió compuestos fenólicos que se encontraban en forma glicosilada y que tienen alta reactividad con el reactivo de Folin-Ciocalteau, conduciendo a un valor elevado de compuestos fenólicos totales (Saikia et al., 2015; Zapata et al., 2015). En este estudio, el valor obtenido para este biocompuesto en el control fue de $41.15 \mathrm{mg} \mathrm{GAE} / 100 \mathrm{~g}$ de materia seca y para el jugo de pitahaya encapsulado, este valor osciló entre 41.92 y $81.53 \mathrm{mg}$ GAE/100 g de materia seca.

Tabla 2: Polifenoles totales contenidos en el control y su comportamiento en función del tiempo de almacenamiento en condiciones ambientales expresados en mg Ac. Gálico/100 g materia seca

\begin{tabular}{|c|c|c|c|c|c|c|}
\hline $\begin{array}{ll}\text { Control } & \text { Tiempo } \\
\end{array}$ & 0 & 2 & 4 & 6 & 24 & $\begin{array}{l}\Delta \text { Polifenoles totales } \\
\text { en el tiempo (\%) }\end{array}$ \\
\hline Jugo de pitahaya amarilla & $41.15 \pm 0.67$ & $39.62 \pm 0.67$ & $38.46 \pm 0.67$ & $35.77 \pm 1.15$ & $28.08 \pm 0.66$ & 31.76 \\
\hline
\end{tabular}


Tabla 3: Polifenoles totales contenidos en los encapsulados y su comportamiento en función del tiempo de almacenamiento tanto en cámara climática (C.C) como en condiciones ambientales (C. Amb.) expresados en mg Ac. Gálico/100 g materia seca

\begin{tabular}{ccccc}
\hline Tratamiento & Tiempo $(\mathrm{h})$ & 0 & $\begin{array}{c}\text { C.C }=\text { C. Amb. } \\
24=96\end{array}$ & $\begin{array}{c}\Delta \text { Polifenoles totales en } \\
\text { el tiempo }(\%)\end{array}$ \\
\hline & & $46.15 \pm 0.08$ & $40.38 \pm 0.22$ & 12.50 \\
& T1 & $41.92 \pm 1.33$ & $22.30 \pm 1.33$ & 46.78 \\
& T3 & $60.57 \pm 1.73$ & $59.23 \pm 0,66$ & 2.22 \\
T4 & $54.23 \pm 1.15$ & $53.07 \pm 0.04$ & 2.12 \\
T5 & $60.57 \pm 0.81$ & $38.07 \pm 0.04$ & 40.00 \\
T6 & $81.53 \pm 0.66$ & $78.46 \pm 0.26$ & 3.77 \\
T7 & $72.69 \pm 1.99$ & $42.69 \pm 0.17$ & 41.26 \\
T8 & $61.73 \pm 2.45$ & $50.76 \pm 0.13$ & 20.48 \\
\hline
\end{tabular}

En el tiempo, se observa una disminución de los polifenoles totales, lo que se puede deber, como lo menciona Pulido et al. (2010), este componente activo es sensible a condiciones ambientales y de procesamiento; sobre todo cuando involucran altas temperaturas, ya que son generalmente termolábiles y aunque tienen diferentes grados de tolerancia al calor, degradación estructural y reordenación, finalmente afectan el contenido total y su actividad (Saikia et al., 2015).

Los encapsulados, presentaron un porcentaje de variación en la concentración de polifenoles entre $2.12 \%$ y $46.78 \%$ en función del tiempo de almacenamiento, encontrándose que la condición de encapsulación a través de secado por atomización que permite la conservación de los polifenoles son las del T4, que, con el paso del tiempo tuvo el menor porcentaje de variación con un $2.12 \%$, seguido de las realizadas con el T3 y T6 con una reducción del 2.22 y $3.77 \%$ respectivamente. Esto pudo ser debido a que los encapsulados obtenidos mediante el T4, posiblemente alcanzaron una temperatura mayor a la de transición vítrea (Tg) y a la de almacenamiento, lo que, según Ramírez-Londoño (2013) contribuye directamente a su estabilidad en almacenamiento. Por otro lado, como lo mencionan Arrazola et al. (2014) este comportamiento también puede ser atribuido a la temperatura de entrada utilizada en el proceso de secado, ya que cuando se emplean temperaturas del aire de entrada bajas, la mayoría de los encapsulados muestran una superficie porosa, rugosa y con hendiduras, que puede incurrir en problemas en sus propiedades de flujo y presentar partículas con grandes áreas de contacto que pueden hacerlas más susceptibles a reacciones de degradación, tales como la oxidación; en cambio cuando la temperatura de entrada al equipo de secado es mayor, se obtiene un mayor número de partículas con superficie lisa, indicando un proceso de microencapsulación más perfecto y más estable. Adicionalmente; se debe tener en cuenta que, la técnica de secado por atomización busca generar un proceso de protección que garantice la conservación de los compuestos y en este caso, el porcentaje de variación en la condición T4 es la que, minimiza el daño que puede estar asociado a temperatura y humedad.

Con la encapsulación mediante secado por aspersión se aumentó de 24 horas para el control hasta 96 horas en los encapsulados, teniendo un incremento de 72 horas en comparación con el control, presentando una alternativa para incrementar la estabilidad en el tiempo de esos biocomponentes. Además, se halló un efecto estadísticamente significativo del tratamiento empleado a través del tiempo, sobre el contenido de polifenoles totales presente tanto en el control como en los encapsulados, con un nivel de significancia del $95 \%$.

\section{Determinación de la capacidad antioxidante.}

Los valores obtenidos de capacidad antioxidante (CA) presente en la pulpa y semilla de pitahaya amarilla (control), así como en los encapsulados, se encuentran entre $75.95 \pm 12.31$ y $101.24 \pm 0.20 \mu \mathrm{gmol} \mathrm{g}^{-1}$ Trolox eq (Tabla 4-7), siendo mayores a los reportados por Beltrán-Orozco et al. (2009) quien evaluó variedades de pitahaya amarilla (16.8 $\mu \mathrm{gmol} \mathrm{g}^{-1}$ Trolox eq), blanca $\left(17.3 \mu \mathrm{gmol} \mathrm{g}{ }^{-1}\right.$ Trolox eq), roja (11 $\mu \mathrm{gmol} \mathrm{g}^{-1} \mathrm{Trolox}$ eq) y cereza (12 $\mu \mathrm{gmol} \mathrm{g} \mathrm{g}^{-1}$ Trolox eq), lo cual se pudo presentar debido a que en esta investigación, se emplearon frutos cultivados en México, razón por la cual puede influir en la composición del fruto, ya que esta, depende de factores como las condiciones climáticas (lluvias, disponibilidad de nutrientes del suelo, variación de la temperatura), el tratamiento del cultivo, la localización geográfica, la aplicación de pesticidas, el estado de la maduración en que fue cosechado, el proceso y almacenamiento al que fue sometido (Mezadri et al., 2006). Estos valores también fueron superiores en comparación al contenido en otras muestras de origen vegetal, tales como: col $\left(17.7 \mu \mathrm{gmol} \mathrm{g}^{-1}\right.$ Trolox eq), fresa (15.4 $\mu \mathrm{gmol} \mathrm{g}^{-1}$ Trolox eq) y espinaca (12.6 $\mathrm{mgmol} \mathrm{g}^{-1}$ Trolox eq); lo que sugiere a esta fruta como potencial para la disminución del riesgo de sufrir enfermedades crónicas; puesto que, como lo menciona Beltrán-Orozco et al. (2009) entre mayor CA posea, mayor será la reducción de la oxidación generada por los radicales libres y menor el deterioro de las células. 
La capacidad antioxidante en los encapsulados, aumentó con respecto al control (jugo de pulpa y semilla) para todos los tratamientos evaluados por la técnica de DPPH y para los tratamientos 1 y 5 evaluados por la técnica de ABTS; lo cual puede estar directamente relacionado al incremento que se presentó en la concentración de polifenoles, ya que entre más alta sea la presencia de compuestos fenólicos, mayor será la capacidad antioxidante (Chaikham et al., 2017). Este comportamiento, puede ser atribuido a que, posiblemente durante el secado como lo mencionan Saikia et al. (2015) y Saikia et al. (2014), se producen reacciones de oxidación que llevan a la oxidación de polifenoles que, en la medición de la capacidad antioxidante muestran valores incluso mayor que la de los polifenoles no oxidados o como lo menciona puede haber presencia de compuestos en el material vegetal distintos a los polifenoles, que contribuyen a aumentar el potencial antioxidante en las muestras. Otra causa como lo menciona Saikia et al. (2014) podría ser, la generación de productos de Maillard, que ocurren a altas temperaturas de proceso; estos productos, pueden actuar como antioxidantes y a su vez, mejorar la actividad, solos o en combinación con otros compuestos fenólicos naturales.

Por otro lado, los resultados de los tratamientos T2, T3, T5, T7 y T8 muestran un comportamiento diferente, presentando valores de capacidad antioxidante determinada por ABTS, menores a los del control; esto puede ser debido conforme a lo reportado por Zapata et al. (2015), a que en el secado por aspersión interviene de forma directa el calor, ocasionando una degradación térmica de los metabolitos antioxidantes que conlleva al rompimiento de grupos químicos funcionales.

Estas diferencias entre tratamientos, pueden ser ocasionadas a que, aunque ambos ensayos miden la capacidad de los antioxidantes presentes en una muestra para capturar el radical libre preformado, sus condiciones de reacción y cinética son diferentes (Tovar-del-Rio, 2013).

Tabla 4: Capacidad antioxidante por ABTS de los encapsulados y su comportamiento en función del tiempo de almacenamiento tanto en cámara climática (C.C) como en condiciones ambientales (C. Amb.) expresada en $\mu g m o l g^{-1}$ Trolox eq

\begin{tabular}{ccccccc}
\hline Tiempo (h) & $\begin{array}{c}\text { C.C C. Amb. } \\
18=72\end{array}$ & $\begin{array}{c}\text { C.C }=\text { C. Amb. } \\
24=96\end{array}$ & $\begin{array}{c}\text { C.C }=\text { C. Amb. } \\
30=120\end{array}$ & $\begin{array}{c}\text { C.C }=\text { C. Amb. } \\
60=240\end{array}$ & $\begin{array}{c}\Delta \text { CA en } \\
\text { el tiempo } \\
(\%)\end{array}$ & $\begin{array}{c}\text { Velocidad global de } \\
\text { descenso } \\
(\mu g m o l ~ g-1 \\
\text { Tratamiento }\end{array}$ \\
\hline T1 & $98.24 \pm 0.75$ & $79.29 \pm 8.31$ & $67.66 \pm 10.30$ & $6.33 \pm 1.50$ & 9.56 & -1.53 \\
T2 & $78.47 \pm 7.82$ & $75.84 \pm 4.42$ & $65.11 \pm 4.27$ & $4.29 \pm 2.99$ & 94.53 & -1.24 \\
T3 & $82.87 \pm 11.76$ & $75.86 \pm 5.13$ & $59.36 \pm 0.29$ & $7.81 \pm 2.45$ & 90.58 & -1.25 \\
T4 & $85.28 \pm 8.48$ & $75.47 \pm 3.33$ & $60.64 \pm 6.09$ & $11.64 \pm 2.47$ & 86.35 & -1.23 \\
T5 & $97.01 \pm 2.63$ & $75.90 \pm 1.94$ & $60.64 \pm 1.19$ & $5.40 \pm 0.75$ & 94.43 & -1.53 \\
T6 & $81.48 \pm 2.68$ & $74.40 \pm 2.55$ & $68.52 \pm 8.91$ & $4.76 \pm 4.29$ & 94.16 & -1.28 \\
T7 & $78.70 \pm 1.48$ & $74.72 \pm 4.64$ & $64.43 \pm 0.59$ & $5.88 \pm 6.69$ & 92.53 & -1.21 \\
T8 & $77.63 \pm 2.54$ & $75.60 \pm 4.44$ & $74.48 \pm 9.82$ & $5.42 \pm 7.10$ & 93.02 & -1.20 \\
\hline
\end{tabular}

Tabla 5: Capacidad antioxidante por ABTS de la pulpa y semilla de pitahaya amarilla y su comportamiento en función del tiempo de almacenamiento en condiciones ambientales expresada en $\mu \mathrm{gmol} \mathrm{g}^{-1}$ Trolox eq

\begin{tabular}{cc}
\hline Tiempo $(h)$ & Pulpa y semilla de pitahaya amarilla (control) \\
\hline 0 & $85.98 \pm 2.87$ \\
0.5 & $66.99 \pm 2.09$ \\
1 & $54.57 \pm 10.53$ \\
1.5 & $36.96 \pm 2.42$ \\
2 & $31.40 \pm 2.75$ \\
2.5 & $28.17 \pm 3.84$ \\
3 & $26.11 \pm 3.57$ \\
3.5 & $22.48 \pm 4.39$ \\
4 & $20.25 \pm 3.18$ \\
4.5 & $13.15 \pm 2.78$ \\
5 & $2.13 \pm 2.66$ \\
$\Delta$ CA en el tiempo $(\%)$ & 97.52 \\
Velocidad global de descenso $\left(\mu \mathrm{gmol} \mathrm{g}{ }^{-1}\right.$ Trolox eq/h) & -16.77 \\
\hline
\end{tabular}


Tabla 6: Capacidad antioxidante por DPPH de los encapsulados y su comportamiento en función del tiempo de almacenamiento tanto en cámara climática (C.C) como en condiciones ambientales (C. Amb.) expresada en $\mu g m o l g^{-1}$ Trolox eq

\begin{tabular}{|c|c|c|c|c|c|c|}
\hline Tiempo $(\mathrm{h})$ & $\begin{array}{c}C \cdot C=C . A m b . \\
18=72\end{array}$ & $\begin{array}{c}\text { C.C = C. Amb. } \\
24=96\end{array}$ & $\begin{array}{c}\text { C.C = C. Amb. } \\
30=120\end{array}$ & $\begin{array}{c}\text { C.C = C. Amb. } \\
60=240\end{array}$ & $\begin{array}{l}\triangle \mathrm{CA} \text { en el } \\
\text { tiempo }(\%)\end{array}$ & $\begin{array}{c}\text { Velocidad global de } \\
\text { descenso } \\
\text { ( } \mu \text { gmol g }{ }^{-1} \text { Trolox eq/h) }\end{array}$ \\
\hline $\mathrm{T} 1$ & $101.20 \pm 0.72$ & $94.18 \pm 4.16$ & $90.64 \pm 4.90$ & $19.50 \pm 0.72$ & 80.73 & -1.36 \\
\hline $\mathrm{T} 2$ & $100.25 \pm 0.37$ & $98.22 \pm 3.41$ & $82.01 \pm 2.37$ & $17.06 \pm 2.65$ & 82.98 & -1.39 \\
\hline T3 & $101.24 \pm 0.20$ & $97.26 \pm 2.93$ & $76.25 \pm 6.16$ & $18.48 \pm 0.45$ & 81.75 & -1.38 \\
\hline T4 & $97.60 \pm 0.53$ & $86.99 \pm 6.47$ & $85,44 \pm 2.17$ & $18.30 \pm 0.77$ & 81.25 & -1.32 \\
\hline T5 & $100.92 \pm 0.23$ & $92.24 \pm 2.83$ & $80.57 \pm 5.04$ & $19.41 \pm 0.56$ & 80.77 & -1.36 \\
\hline T6 & $99.17 \pm 0.04$ & $96.25 \pm 2.45$ & $83.70 \pm 3.17$ & $19.33 \pm 0.46$ & 80.51 & -1.33 \\
\hline T7 & $98.76 \pm 0.67$ & $97.32 \pm 2.28$ & $77.27 \pm 810.21$ & $18.34 \pm 0.57$ & 81.43 & -1.34 \\
\hline T8 & $97.85 \pm 0.30$ & $97.16 \pm 1.25$ & $91.74 \pm 4.59$ & $17.61 \pm 0.92$ & 82.00 & -1.34 \\
\hline
\end{tabular}

Tabla 7: Capacidad antioxidante por DPPH de la pulpa y semilla de pitahaya amarilla y su comportamiento en función del tiempo de almacenamiento en condiciones ambientales expresada en $\mu \mathrm{gmol} \mathrm{g}^{-1} \mathrm{Trolox}^{\mathrm{eq}}$

\begin{tabular}{cc}
\hline Tiempo $(\mathrm{h})$ & Pulpa y semilla de pitahaya amarilla (control) \\
\hline 0 & $75.95 \pm 12.31$ \\
0.5 & $66.98 \pm 5.51$ \\
1 & $52.31 \pm 11.71$ \\
2 & $59.01 \pm 3.24$ \\
2.5 & $45.46 \pm 10.12$ \\
3 & $38.94 \pm 1.00$ \\
3.5 & $38.15 \pm 4.73$ \\
4 & $30.10 \pm 3.48$ \\
4.5 & $19.69 \pm 0.75$ \\
5 & $14.40 \pm 4.43$ \\
Velocidad global de descenso $\left(\mu \mathrm{gmol} \mathrm{g}^{-1}\right.$ Trolox eq/h) & $8.75 \pm 0.54$ \\
\hline
\end{tabular}

La capacidad antioxidante del control y los encapsulados va decreciendo en función del tiempo de almacenamiento para los dos métodos, ABTS (Tabla 4 y 5) y DPPH (Tabla 6 y 7); no obstante, se presenta una prolongación del tiempo de vida útil con la encapsulación a través de secado por atomización, logrando pasar de 5 horas para el control hasta 240 horas en los encapsulados, teniendo un incremento de 235 horas con respecto al control. La velocidad de descenso calculada mediante la Ecuación 1, no mostró diferencias significativas con un nivel de confianza del 95\% entre las técnicas ABTS y DPPH, ni entre los tratamientos. Sin embargo, teniendo en cuenta la prueba de comparación de Tukey, se puede establecer el T4 como aquel que conservó la capacidad antioxidante de los biocompuestos en el tiempo; lo cual coincide con los resultados obtenidos en polifenoles, atribuyendo este comportamiento igualmente a que los encapsulados obtenidos mediante el T4, posiblemente alcanzaron una temperatura mayor a la de transición vítrea (Tg) y a la de almacenamiento, lo que, según Ramírez-Londoño (2013) contribuye directamente a su estabilidad.

Por otro lado, como lo mencionan Arrazola et al. (2014) esto también puede ser debido a la temperatura de entrada utilizada en el proceso de secado, ya que cuando se emplean temperaturas del aire de entrada bajas, la mayoría de los encapsulados muestran una superficie porosa, rugosa y con hendiduras, que puede incurrir en problemas en sus propiedades de flujo y presentar partículas con grandes áreas de contacto que pueden hacerlas más susceptibles a reacciones de degradación, tales como la oxidación; en cambio cuando la temperatura de entrada al equipo de secado es mayor, se obtiene un mayor número de partículas con superficie lisa, indicando un proceso de microencapsulación más perfecto y más estable.

\section{Simulación de la liberación in vitro de los biocomponentes de la pitahaya amarilla encapsulada}

La simulación de la liberación in vitro llevada a cabo, demuestra que, aunque en la fase estomacal, se presentó una liberación de polifenoles totales contenidos en los encapsulados de 5.9 a $11.9 \mathrm{mg}$ GAE/ $100 \mathrm{~g}$ de materia seca (Fig. 1), estos consiguen resistir las condiciones de pH 2 durante las 4 horas en las que el encapsulado por ser un material sólido permanece en tal fase, logrando finalmente que en la fase intestinal en la cual los 
encapsulados entran en contacto con las sales biliares y un $\mathrm{pH}$ de 7 , se lleve a cabo la liberación de la mayoría del biocomponente (Fig. 2), quedando disponible para su adsorción y cumplir así con su función biológica (Fernández-León, 2015).

La diferencia entre el proceso desortivo a nivel estomacal e intestinal puede ser debido a que la mayoría de los compuestos fenólicos están presentes en los alimentos como esteres, glicósidos o polimeros, de los cuales, los glicósidos resisten la hidrolisis ácida del estómago y llegan intactos al intestino en donde son metabolizados por efecto de las enzimas intestinales y la alcalinidad del medio (Arenas et al., 2017), indicando esto último que los fenoles son inestables en medio neutro o alcalino.

Dicha conducta también puede estar relacionada con el agente encapsulante en el que se encuentran los compuestos fenólicos ya que este, posiblemente, ejerce una barrera protectora que se va deteriorando a medida que los encapsulados están en contacto con el medio ácido y las enzimas de la fase estomacal simulada, las cuales pueden actuar sobre compuestos con alto peso molecular como los carbohidratos que contiene el material de soporte (maltodextrina), liberando los fenoles y aumentando de este modo la concentración de estos compuestos con el paso del tiempo (da Silva Fernandes et al., 2017).

Los valores concernientes a la liberación de los compuestos fenólicos presentaron diferencias estadísticamente significativas con un nivel de confianza del $95 \%$. Se encontró, además que el tratamiento que presenta mayor resistencia a las condiciones en la fase estomacal, es el tratamiento 6 que fue llevado a cabo a una temperatura de secado de $120^{\circ} \mathrm{C}$ y una concentración de agente encapsulante del $50 \%$ con una liberación equivalente al $34.2 \%$, seguido del tratamiento $3\left(130{ }^{\circ} \mathrm{C} / 40 \%\right)$ con una liberación del $51.8 \%$. Ya en la fase intestinal, el tratamiento que presenta mayor liberación de polifenoles es el $4\left(150{ }^{\circ} \mathrm{C} / 40 \%\right)$ con una liberación equivalente al $90.8 \%$, seguido del tratamiento $6\left(120^{\circ} \mathrm{C} / 50 \%\right)$ y el tratamiento $2\left(120^{\circ} \mathrm{C} / 40 \%\right)$ con liberaciones del 90.2 y $90.1 \%$.

El comportamiento diferente en la liberación de los compuestos fenólicos de las muestras puede ser atribuido a factores, como las condiciones de procesamiento del producto (temperatura) y la concentración de la matriz portadora (da Silva Fernandes et al., 2017; Rodríguez-Roque et al., 2013). Altas temperaturas de secado, por ejemplo, incurren en la obtención de encapsulados con paredes agrietadas a través de las cuales probablemente se pueda generar la liberación de compuestos fenólicos (Ramírez-Londoño, 2013). Por otro lado, las interacciones entre estos compuestos y otros constituyentes, pueden favorecer la formación de complejos con baja solubilidad o con alto peso molecular, que no pueden atravesar la membrana, causando una reducción en la concentración fenólica (Rodríguez-Roque et al., 2013).

A

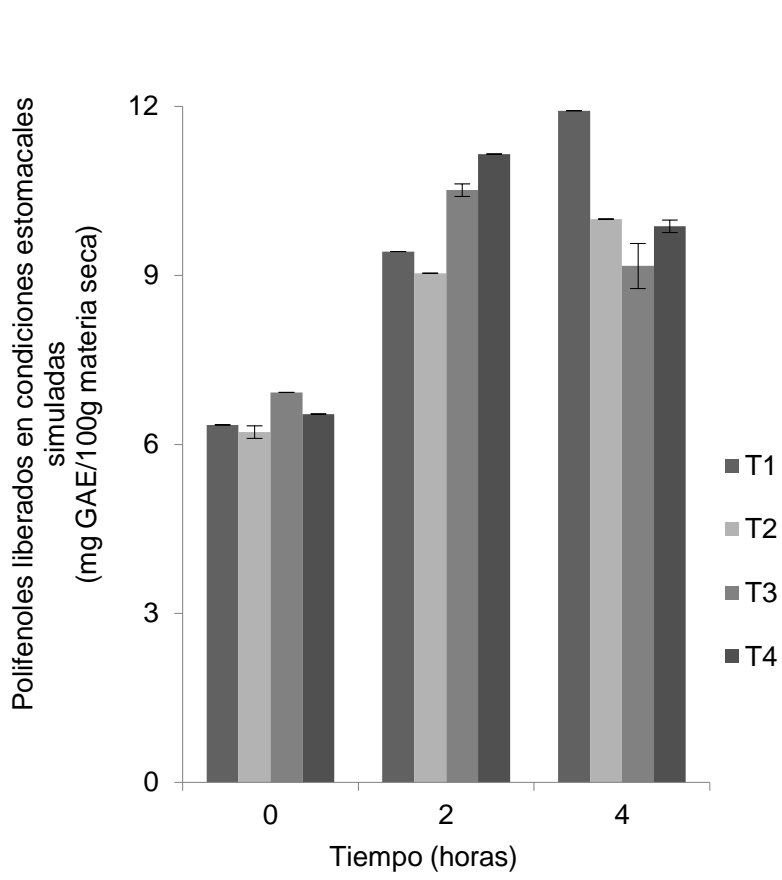

B

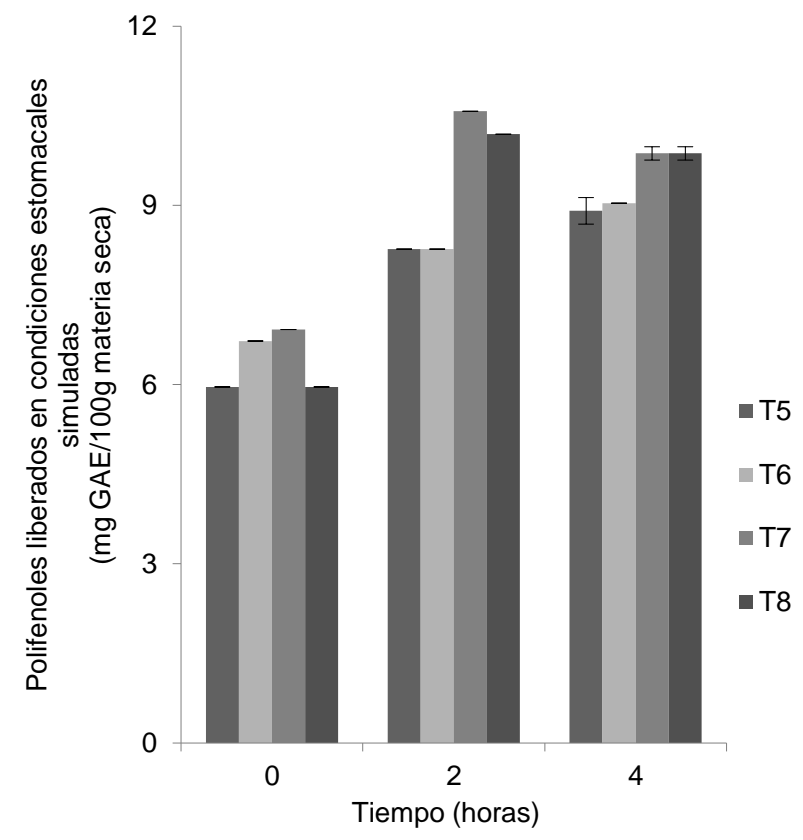

Fig. 1: Liberación in vitro de polifenoles totales de los encapsulados sometidos durante 4 horas a la fase estomacal simulada. Dónde A: concentración de maltodextrina al 40\%, B: Concentración al 50\% 
A

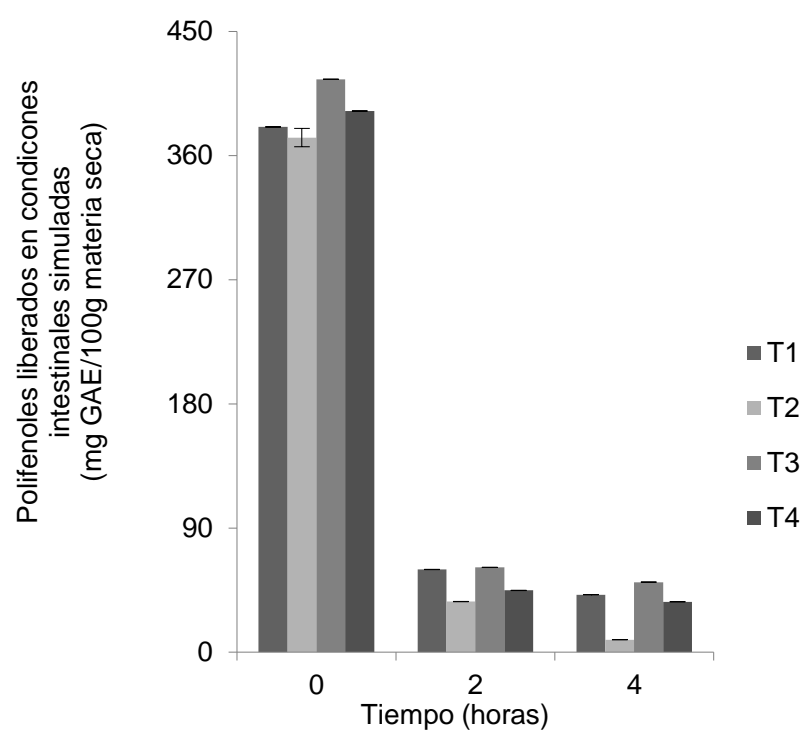

B

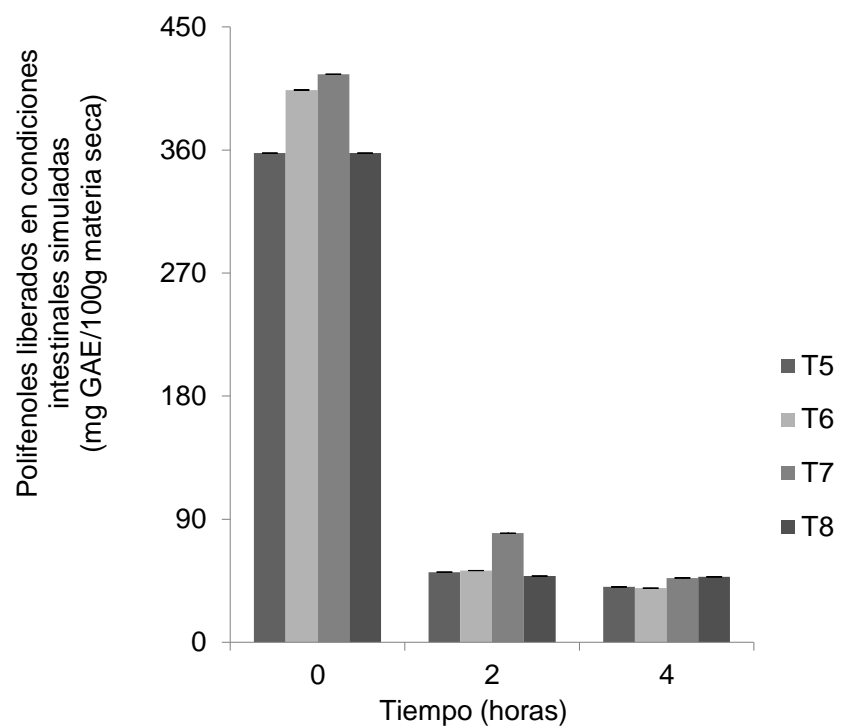

Fig. 2: Liberación in vitro de polifenoles totales de los encapsulados sometidos durante 4 horas a la fase intestinal simulada. Dónde A: concentración de maltodextrina al 40\%,B: Concentración al 50\%

\section{CONCLUSIONES}

A partir de los parámetros analizados durante esta investigación, se infiere que el tratamiento que tuvo un mayor efecto en la conservación de los biocomponentes funcionales de la mezcla de pitahaya amarilla (pulpa y semilla), fue aquel que se llevó a cabo a una temperatura de $150^{\circ} \mathrm{C}$ con $40 \%$ agente encapsulante (T4), el cual además de prolongar la estabilidad hasta 72 horas para polifenoles totales y 235 horas para la capacidad antioxidante, también garantizó que la liberación de los mismos se realice en el intestino delgado que es donde deben quedar disponibles para que el organismo pueda llegar a absorberlos y logren cumplir con su función.

La estabilidad de los componentes bioactivos contenidos en la pulpa y semilla de pitahaya amarilla, se prologaron 100 veces más con respecto al control, esto, mediante la encapsulación a través del secado por atomización; por lo cual se puede considerar técnica viable para la conservación de biocomponentes funcionales siempre y cuando se empleen concentraciones del agente encapsulante y temperaturas de secado óptimas, consiguiendo además, mejorar la biodisponibilidad lo que convierte este proceso, en una alternativa para la generación de productos con alto valor agregado que permitan aumentar la estabilidad en almacenamiento, puesto que para los biocomponentes (polifenoles y antioxidantes) de esta fruta en fresco, dicha estabilidad es corta en comparación a si se es sometida a técnicas de conservación como la encapsulación.

\section{AGRADECIMIENTOS}

Los autores expresan sus agradecimientos al departamento administrativo de ciencia, tecnología e información COLCIENCIAS, por la financiación del proyecto.

\section{REFERENCIAS}

Araujo-Díaz, S.B., C. Leyva-Porras, P. Aguirre-Bañuelos, C. Álvarez-Salas y Z. Saavedra-Leos, Evaluation of the physical properties and conservation of the antioxidants content, employing inulin and maltodextrin in the spray drying of blueberry juice, doi: https://doi.org/10.1016/j.carbpol.2017.03.065, Carbohydr Polym. (en línea), 167, 317-325 (2017)

Arenas, E.H. y T.P. Trinidad, Fate of polyphenols in pili (Canarium ovatum Engl.) pomace after in vitro simulated digestion, doi: https://doi.org/10.1016/j.apjtb.2016.11.002, Asian Pac. J. Trop. Biomed. (en línea), 7(1), 53-58 (2017)

Arrazola, G., I. Herazo y A. Alvis, Microencapsulación de Antocianinas de Berenjena (Solanum melongena L.) mediante Secado por Aspersión y Evaluación de la Estabilidad de su Color y Capacidad Antioxidante, doi: http://dx.doi.org/10.4067/S0718-07642014000300006 Inf. Tecnol. (en línea), 25, 31-42 (2014) 
Beltrán-Orozco, M.C., T.G. Oliva-Coba, T. Gallardo-Velázquez y G. Osorio-Revilla, Ascorbic acid, phenolic content, and antioxidant capacity of red, cherry, yellow and white types of pitaya cactus fruit (Stenocereus stellatus Riccobono), Agrociencia. 43, 153-162 (2009)

Borgogna, M., B. Bellich, L. Zorzin, R. Lapasin y A. Cesàro, Food microencapsulation of bioactive compounds: Rheological and thermal characterisation of non-conventional gelling system, doi: http://dx.doi.org/10.1016/j.foodchem.2009.07.043, Food Chemistry. (Online), 122(2), 416-423 (2010)

Carlan, I.C., B.N. Estevinho y F. Rocha, Study of microencapsulation and controlled release of modified chitosan microparticles containing vitamin B12, doi: https://doi.org/10.1016/j.powtec.2017.05.041, Powder Technol. (en línea), 318, 162-169 (2017)

Chaikham, P., V. Kemsawasd y P. Seesuriyachan, Spray drying probiotics along with maoluang juice plus Tiliacora triandra gum for exposure to the in vitro gastrointestinal environments, doi: https://doi.org/10.1016/j.lwt.2016.12.013, LWT - Food Sci Technol. (en línea), 78, $31-40$ (2017)

da Silva Fernandes, M., F. Sanches Lima, D. Rodrigues, C. Handa, M. Guelfi, S. Garcia y E.I. Ida, Evaluation of the isoflavone and total phenolic contents of kefir-fermented soymilk storage and after the in vitro digestive system simulation, doi: https://doi.org/10.1016/j.foodchem.2017.02.095, Food Chem. (en línea), 229, 373-380 (2017)

Díaz-Martínez, Y.L., J.A. Serna-Jiménez, L.S. Torres-Valenzuela y J.L. Hoyos-Concha, Determinación de la temperatura de secado para la encapsulación de proteasa mediante secado por atomización, Ingenio Magno, ISSN: 2422-2399 (en línea), 7(1), 134-142, 2016. https://goo.gl/gXgyDW, Acceso: Junio (2017)

Ee, S.C., B. Jamilah, K. Muhammad, D.M. Hashim y N. Adzahan, Physico-chemical properties of spray-dried red pitaya (Hylocereus polyrhizus) peel powder during storage, International Food Research Journal, ISSN: 1985-4668 (Online), 21(1), 155-160 (2014)

Fernández-León, A.M., Estudio de la biodisponibilidad y bioactividad de los compuestos funcionales presentes en brócoli y col de milán. (Tesis Doctoral), Universidad de Extremadura. Departamento de Biología Vegetal, Ecología y Ciencias de la Tierra (2015)

Jara-Palacios, M.J., D. Hernanz, S. González-Manzano, C. Santos-Buelga, M.L. Escuderi-Gilete y F.J. Heredia, Detailed phenolic composition of white grape by-products by RRLC/MS and measurement of the antioxidant activity, doi: http://dx.doi.org/10.1016/j.talanta.2014.02.065, Talanta. (Online), 125, 51-57 (2014)

Kar-Hing, L., W. Ta-Yeong y S. Lee-Fong, Spray drying of red (Hylocereus polyrhizus) and white (Hylocereus undatus) dragon fruit juices: physicochemical and antioxidant properties of the powder, doi: 10.1111/ijfs.12230, International Journal of Food Science and Technology. (Online), 4(1), 2391 - 2399 (2013)

Matsuura, T., A. Ogawa, M. Tomabechi, R. Matsushita, S. Gohtani, T.L. Neoh y H. Yoshii, Effect of dextrose equivalent of maltodextrin on the stability of emulsified coconut-oil in spray-dried powder, doi: http://dx.doi.org/10.1016/j.jfoodeng.2015.04.018, Journal of Food Engineering. (Online), 163, 54-59 (2015)

Mezadri, T., M.S. Fernández-Pachón, D. Villaño, M.C. García-Parrilla y A.M. Troncoso, El fruto de la acerola: composición y posibles usos alimenticios, Archivos Latinoamericanos de Nutrición, ISSN: 0004-0622 (en línea), 56(2), 101-109, 2006. https://goo.gl/yTcrDz, Acceso: Mayo (2015)

Porras-Saavedra, J., E. Palacios-González, L. Lartundo-Rojas, V. Garibay-Febles, J. Yáñez-Fernández, H. Hernández-Sánchez, G.Gutiérrez-López, L. Alamilla-Beltrán, Microstructural properties and distribution of components in microparticles obtained by spray-drying, doi: http://dx.doi.org/10.1016/j.jfoodeng.2014.11.014, Journal of Food Engineering. (Online), 152(0), 105-112 (2015)

Posada-Cardona, C.C., Recopilación de estudios de tiempos de vida útil de productos nuevos y ya existentes de la compañia de galletas NOEL S.A.S. (Tesis de pregrado), Corporación Universitaria Lasallista. Facultad de Ingeniería (2011)

Pulido, A. y C.I. Bristain, Encapsulación de ácido ascórbico mediante secado por aspersión, utilizando quitosano como material de pared, Revista Mexicana de Ingeniería Química, 1665-2738 (en línea), 9, 189195, 2010. https://goo.gl/SSWQgZ, Acceso: Mayo (2015)

Ramírez-Londoño, M.J., Evaluación de variables de un proceso de micro-encapsulación para la estabilización de polifenoles. (Tesis de Maestría), U. Nacional de Colombia, Manizales, Colombia. Depto. de Ing. Química (2013) 
Ramírez, M.J., N. Salgado-Aristizabal y C.E. Orrego-Alzate, Conservación de polifenoles en un jugo de fruta modelo secado por aspersión y liofilización, Vitae, 0121-4004 (en línea), 19(1), S87-S89, 2012. https://goo.gl/VDq3AZ, Acceso: Mayo (2015)

Rodríguez-Roque, M.J., M.A. Rojas-Graü, P. Elez-Martínez y O. Martín-Belloso, Soymilk phenolic compounds, isoflavones and antioxidant activity as affected by in vitro gastrointestinal digestion, doi: https://doi.org/10.1016/j.foodchem.2012.07.115, Food Chem. (en línea), 136(1), 206-212 (2013)

Saikia, S., N. Mahnot y C. Mahanta, Effect of Spray Drying of Four Fruit Juices on Physicochemical, Phytochemical and Antioxidant Properties, doi: 10.1111/jfpp.12395, J. Food Process. Preserv. (en línea), 39(6), 1-9 (2014)

Saikia, S., N.K. Mahnot y C.L. Mahanta, Optimisation of phenolic extraction from Averrhoa carambola pomace by response surface methodology and its microencapsulation by spray and freeze drying, doi: https://doi.org/10.1016/j.foodchem.2014.08.064, Food Chem. (en línea), 171, 144-152 (2015)

Shaaruddin, S., H.M. Ghazali, S. Hamed Mirhosseini y K. Muhammad, Stability of betanin in pitaya powder and confection as affected by resistant maltodextrin, doi: https://doi.org/10.1016/j.Iwt.2017.05.031, LWT Food Sci. Technol. (en línea), 84, 129-134 (2017)

Sharifi, A., M. Niakousari, A. Maskooki y S. Mortazavi, Effect of spray drying conditions on the physicochemical properties of barberry (Berberis vulgaris) extract powder, International Food Research Journal, 2364-2370 (Online), 22(6), 2364-2370 (2015)

Shete, C.C., S.S. Wadkar, N.B. Gaikwad, K.S. Patil y J.S. Ghosh, Phenolic contents and antioxidant capacity of Amorphophallus commutatus and Amorphophallus paeonifolius, International Food Research Journal, 1939-1944 (Online), 22(5), 1939-1944 (2015)

Souza, V.B.d., A. Fujita, M. Thomazini, E.R. da Silva, J.F. Lucon Jr, M.I. Genovese y C.S. Favaro-Trindade, Functional properties and stability of spray-dried pigments from Bordo grape (Vitis labrusca) winemaking pomace, doi: https://doi.org/10.1016/j.foodchem.2014.05.049, Food Chem. (en línea), 164, 380-386 (2014)

Tovar-del-Rio, J. Determinación de la actividad antioxidante por DPPH y ABTS de 30 plantas recolectadas en la ecoregion cafetera. (Tesis de Pregrado), Universidad Tecnológica de Pereira, Pereira. Escuela de Técnología Química (2013)

WHO. Global status report on noncommunicable diseases 2014. Online. WHO Library Cataloguing-inPublication Data. (2014)

Zapata, K., B.A. Rojano y F.B. Cortes, Efecto Térmico del Secado por Aspersión sobre los Metabolitos Antioxidantes de la Curuba Larga (Passiflora mollisima baley), doi: 10.4067/S0718-07642015000100009, Información Tecnológica. (en línea), 26(1), 77-84 (2015) 